\title{
Extra-mucosal laryngectomy for the treatment of radiation-induced non-functional larynx
}

\author{
Salim Bouayed, Andrea Avagnina and Bassel Hallak* \\ Department of Otorhinolaryngology, Head and Neck Surgery, Hospital of Sion, Sion, Switzerland
}

\begin{abstract}
Background: Total laryngectomy is a very commonly performed procedure for treating patients with non-functional larynx after (chemo) radiotherapy. Postoperative fistula is the major and most-feared complication. We would like to outline our experience regarding a new surgical approach by extra-mucosal laryngectomy with the aim of conserving the totality of the pharyngeal mucosa thereby reducing the incidence of port-operative pharyngo-cutaneous fistula.

Case presentation: We report the case of a 54-year old, female Caucasian who presented with bilateral, progressive recurrent and hypoglossal nerve paralysis four years after undergoing chemo-radiotherapy for carcinoma of the larynx in 2008. She had a major functional laryngeal disorder with absence of improvement despite several therapies, reason why we performed a new approach of an extra-mucosal laryngectomy. The operative technique and clinical outcomes are documented here.

Conclusion: Extra-mucosal laryngectomy provided a good surgical option for the treatment of a nonfunctional larynx. The procedure is simple and allows for total conservation of the pharyngeal mucosa with the main advantage being the absence of post-operative fistula.
\end{abstract}

\section{Introduction}

The normal human larynx has three major functions: phonation, breathing and airway protection. The voice is not essential for survival and sometimes could be compromised in an effort to preserve the airway and prevent aspiration [1]. Chronic repetitive bronchoaspiration is a serious consequence of the nonfunctional larynx, with increased morbidity and mortality. The treatment of aspiration comprises; conservative management and surgical procedures which can be divided into adjuvant and definitive procedures [2]. The adjuvant procedures include tracheotomy, cricopharyngeal myotomy [3], laryngeal suspension, cricoid resection [4] and various vocal fold medializations.

When the adjuvant procedures fail to manage the aspiration and the patient presents intractable life-threatening aspiration, procedures that are more definitive should be applied. These consist of various techniques for blocking or separating the larynx and the trachea; they include laryngeal stenting, glottic and supraglottic laryngeal closure [5].

Total laryngectomy and tracheal diversion are the most definitive performed procedures to manage patients with nonfunctional larynx and chronic aspiration. Pharyngocutaneous fistula (PCF) represents the most common and troublesome postoperative complication with an incidence rate in the literature ranging from $5 \%$ to $65 \%$ [6].

We report a case of nonfunctional larynx-induced chronic aspiration complicated by bilateral lingual paralysis. Multiple procedures to manage the aspiration were undertaken without success. Extra-mucosal laryngectomy was performed in order to maximize conservation of the mucosa. The surgical procedure and clinical outcomes are presented here.

\section{Case presentation}

A 54-year-old, female Caucasian was diagnosed in 2008 for squamous cell carcinoma of the supraglottic larynx and unilateral left sided vocal fold immobility, initially staged as cT3cN1cM0. She underwent chemo-radiotherapy (total dose of 70 Gy combined with 3 cycles of Cisplatin $100 \mathrm{mg} / \mathrm{m}^{2}$ ). Her medical background did not reveal any other problems. The clinical and radiological follow-up showed a total remission without evidence of recurrence of her oncological disease.

She presented three years later, in 2011, with acute dyspnea and inspiratory stridor. The fibro-endoscopic examination of the larynx showed a paralysis of the vocal fold on the right side in addition to the paralysis of the left vocal fold already present. This bilateral paralysis of the vocal folds in a para-median position induced significant narrowing of the larynx airway. There was no evidence of tumor recurrence. An emergency tracheotomy was performed. The etiology of the paralysis of the right vocal fold on the opposite side of the primary laryngeal tumor was considered as radiation-induced recurrent nerve paralysis. In a second step, a unilateral arytenoidectomy combined with posterior cordectomy on the right vocal fold was performed with closure of the tracheostomy. The follow-up showed no recurrence of acute dyspnea but exercise-induced difficulties in breathing combined with swallowing disorders and aspiration were present.

Thereafter, in 2012, she developed a unilateral paralysis of the tongue on the left-hand side which worsened the swallowing issues rendering the situation quite critical. This was followed in 2014, by

${ }^{*}$ Correspondence to: Bassel Hallak, Department of Otorhinolaryngology, Hospital of Sion, Avenue du Grand-Champsec 80, 1950 Sion, Switzerland, Email: bassel2004@hotmail.com, bassel.hallak@hopitalvs.ch

Key words: larynx, radiotherapy, functional disorders, chronic aspiration, surgical technique

Received: September 14, 2020; Accepted: September 21, 2020; Published: September 28,2020 
the paralysis of the remaining right-hand side of the tongue. The oral ingestion became impossible and the patient was feeding exclusively through a gastrostomy tube.

The follow-up showed a deterioration of the chronic aspiration with, in 2017, the occurrence of bilateral pneumonia complicated by sepsis requiring intensive care management with antibiotic therapy. The oncological outcomes continued to show absence of recurrence or distant metastasis.

Due to the persistence and pejoration of chronic aspiration with the incidence of complicated pneumonia, inability of oral ingestion and failure of all the therapies applied to manage this situation, the decision to perform a total laryngectomy as a definitive procedure was taken. In order to reduce as much as possible the incidence of post-operative fistula, an extra-mucosal laryngectomy was performed. The principal of this new surgical procedure is to conserve the totality of the pharyngeal mucosa and avoid any opening of the hypopharyngeal tube.

The procedure was performed by a trans-cervical approach. The skin incision ( $4 \mathrm{~cm}$ in length) was performed at the level of the cricothyroid membrane (Figure1a), followed by sub-cutaneous dissection and spacing of the underlying muscles. The pre-laryngeal musculature is incised on the medial line and lifted down to the first tracheal ring inferiorly and up superiorly, to opposite to the crico-thyroid membrane. The larynx is thus exposed (Figure1b). Tracheostomy was performed at the level of the second tracheal ring.

The extra-mucosal laryngectomy begins with detachment of the external perichondrial thyroid wings to the posterior edge, followed by median thyrotomy without opening the laryngeal mucosa. Detachment of the posterior surface of the thyroid cartilage at the level of its posterior edge. The upper and lower thyroid processes were sectioned at their base and left in place. The thyroid wings were removed (Figures $1 \mathrm{c}$ and $1 \mathrm{~d}$ ). The cricoid ring was sectioned anteriorly and then retracted up to expose the posterior surface, in order to detach progressively the hypopharyngeal mucosa at the level of the superior edge of the cricoid cartilage. The cricoid cartilage was totally removed and the remaining arytenoid was left untouched (Figure 2a). Thus, one obtained a laryngeal.

Mucosal ring which was then sutured at the level of both ventricular bands in 2 layers using resorbable sutures (maxon 4/0 and vicryl $4 / 0$ slow resorption) (Figures $2 \mathrm{~b}$ and $2 \mathrm{c}$ ). An aspirating drain is placed, and the skin was closed classically (Figure $2 \mathrm{~d}$ ).

The post-operative care was simple, without complications. The drain was removed 2 days later, and feeding was continued by the gastrostomy tube. The patient was discharged 5 days after the surgery.

Three weeks post-operatively, the patient developed a dermohypodermitis near the cervical scar, successfully treated by local antibiotic ointment. She benefited from physiotherapy swallowing sessions with progressive introduction of oral feeding, starting initially with liquids at three weeks post-operatively. Six months later, the patient was able to eat chopped and even small pieces of solids foods. Fibro-endoscopic examination showed good healing of the pharyngeal mucosa with mild narrowing of the lumen.

The follow-up at 10 months showed progressive and continued subjective improvement of oral feeding.
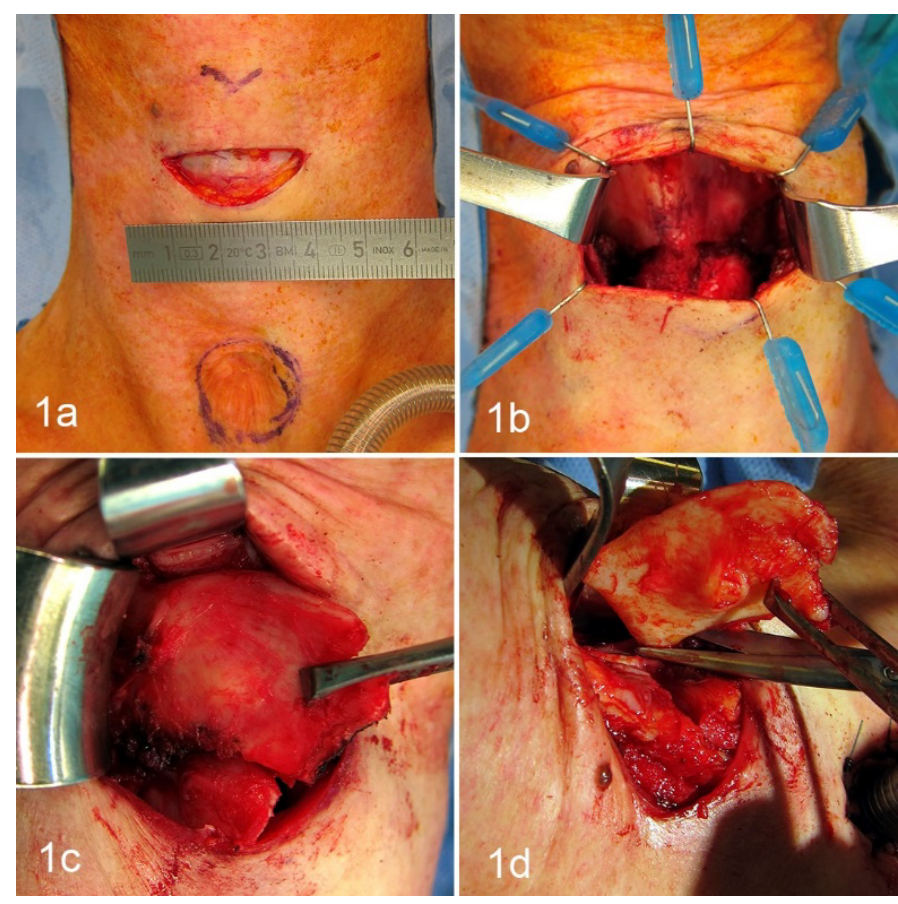

Figure 1. a: skin incision at the level of the crico-thyroid membrane, b: exposure of the laryngeal cartilages, c: removing of the right thyroid wing, d. removing of the left thyroid wing

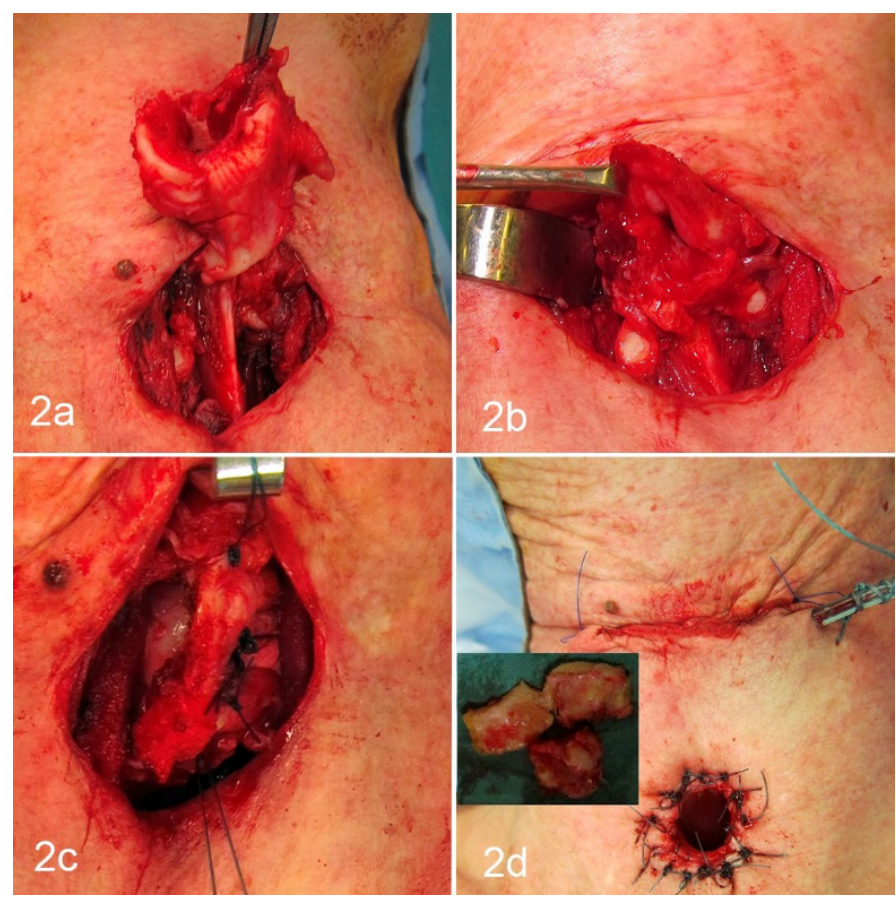

Figure 2. a: removing of the cricoid cartilage, b: exposure of the laryngeal mucosal tube, c: sutures of the laryngeal mucosal tube, d: skin sutures and closure of the cervical wound

\section{Discussion}

Aspiration can occur in patients with various pathological processes. It is very important to determine the underlying etiology as this directs the treatment options. Several studies have shown the degradation of laryngeal functions after chemo-radiotherapy, especially 
swallowing. It results in a decrease in larynx elevation, retraction of the base of the tongue, and pharyngeal contraction $[7,8]$.

Multiple surgical procedures have been described to treat aspiration problems with the aim of reducing the risk of postoperative fistula. Tracheal diversion has the advantage of leaving the larynx in place, resulting in a lower risk of complication [9]. This procedure is surgically difficult due to the terrain (post-radiation neck) and the quality of the trachea in cases of previous tracheotomy.

Dulguerov et al. reported the Open Maximum Mucosa-Sparing Functional Total Laryngectomy with a fistula rate of $10 \%$. The main principals of the extra-mucosal laryngectomy as described above are the absence of opening of the hypopharynx with total conservation of the mucosa and the closure of the laryngeal tube obtained. This allows an important decrease in the incidence of fistulas. The surgical procedure is simple and carries a lot of advantages in term of postoperative care; short period of hospitalization, possibility of starting swallowing rehabilitation quite quickly and especially the absence of post-operative fistula or other serious complications.

Our patient presented progressive bilateral recurrent and hypoglossal nerves paralysis after undergoing radiotherapy and this situation could be typically compatible with a Tapia syndrome. The follow-up at 10 months showed the absence of fistula formation and improvement in oral ingestion. Regarding the voice, the patient refused our proposition for phonatory prosthesis. She preferred to follow speech therapy (esophageal voice).

\section{Conclusion}

1. Radiation-induced nonfunctional larynx and chronic aspiration is problematic with an important impact on the prognosis and the quality of life.

2. Total laryngectomy as a definitive surgical procedure caries the risk of fistula, especially in irradiated necks.
3. Extra-mucosal laryngectomy allows total conservation of the pharyngeal mucosa where the main advantage is to reduce the incidence of fistula.

4. The surgical procedure is simple, rapid to perform, without complications and caries no risk of post-operative fistula.

5. This technique is a good option to treat successfully repetitive aspirations.

\section{References}

1. Desheng W, Pavel D (2000) Laryngeal diversion and tracheotracheal speech fistula for chronic aspiration. Ann Otol Rhinol Laryngol 109: 15. [Crossref]

2. Miller FR, Eliachar I (1994) Managing the aspiration patient. Am J Otolaryngol 15:117.

3. Sasaki CT, Suzuki M, Horiuchi M, Kirchner JA (1977) The effect of tracheostomy on the laryngeal closure reflex. Laryngoscope 87:1428-1433. [Crossref]

4. Krespi YP, Pelzer HJ, Sisson GA (1985) Management of cheonic aspiration by subtotal and submucosal cricoid resection. Ann Otol Rhinol Laryngol 94: 580-583. [Crossref]

5. Montgomery WW (1975) Surgical laryngeal closure to eliminate chronic aspiration. $N$ Engl J Med 292: 1390-1391.

6. Aprigaliano E, Levine H (1997) Pharyngeal reconstruction after laryngectomy. Laryngoscope 87:1884-1890. [Crossref]

7. Pauloski BR, Rademaker AW, Logemann JA, Newman L, MacCracken E, et al. (2006) Relationship between swallow motility disorders on videofluorography and oral intake in patients treated for head and neck cancer with radiotherapy with or without chemotherapy. Head Neck 28:1069-1076. [Crossref]

8. Kraaijenja SA, Oskam IM, Van der Molen L, Hamming-Vrieze O, Hilgers FJ, et al (2015) Evaluation of long term (10-years + ) dysphagia and trismus in patients treated with concurrent chemo-radiotherapy for advanced head and neck cancer. Oral Oncol 51: 787-794. [Crossref]

9. Adachi K, Umezaki T, Kiyohara H, Miyaji H, Komune S (2015) Clinical outcomes of tracheoesophageal diversion for intractable aspiration. J Laryngol Otol 129: S69-73. [Crossref]

Copyright: (C2020 Bouayed S. This is an open-access article distributed under the terms of the Creative Commons Attribution License, which permits unrestricted use, distribution, and reproduction in any medium, provided the original author and source are credited. 